CARDIOVASCULAR MEDICINE

\title{
Variable left ventricular activation pattern in patients with heart failure and left bundle branch block
}

\author{
J W-H Fung, C-M Yu, G Yip, Y Zhang, H Chan, C-C Kum, J E Sanderson
}

Heart 2004;90:17-19

\begin{abstract}
See end of article for authors' affiliations

.....................

Correspondence to: Dr J W-H Fung, Division of Cardiology, Department of Medicine and Therapeutics, Prince of Wales Hospital, The Chinese University of Hong Kong, Hong Kong, SAR, China; jwhtung@ cuhk.edu.hk
\end{abstract}

Accepted 11 June 2003

\begin{abstract}
Objective: To determine the left ventricular (LV) activation pattern in patients with chronic heart failure and left bundle branch block (LBBB) on ECG.

Design: Prospective study.

Setting: Tertiary cardiology referral centre in Hong Kong.

Patients: Seven patients with LV ejection fraction $<35 \%$ and typical LBBB on ECG with QRS duration $\geqslant 130 \mathrm{~ms}$ were recruited. Five of them had non-ischaemic dilated cardiomyopathy.

Methods: Non-contact mapping was used to investigate the LV global activation sequences. Tissue Doppler imaging was performed with the LV mapping and correlated with the activation sequences.

Results: Three patients had preserved left bundle activation despite LBBB on ECG. Conduction block was detected in four patients during LV activation and the other three had homogeneous depolarisation propagation within the left ventricle. The latest segment of activation was located in either the lateral or the posterior region. Tissue Doppler imaging correlated well with non-contact mapping to locate the conduction block and the latest segment of activation.

Conclusions: LV endocardial activation sequences in patients with chronic heart failure and LBBB are variable. This may have implications for patient selection for treatment with cardiac resynchronisation.
\end{abstract}

\section{METHODS}

Seven patients with New York Heart Association class III with ejection fraction $<35 \%$ and LBBB morphology with QRS duration $\geqslant 130 \mathrm{~ms}$ were studied. The mean (SD) age was 66.1 (7.3) years, QRS duration was 152 (19) ms, and ejection fraction was $23(7) \%$. Five patients had nonischaemic heart failure. All patients were in sinus rhythm and received optimal medical treatment. Written informed consent was obtained from all patients. LV activation was mapped and TDI assessed simultaneously.

The LV activation pattern was delineated by non-contact mapping as previously described. ${ }^{4}$ The left ventricle was divided into basal, mid, and apical sections in a longitudinal axis. Each section was divided further into anterior, posterior, lateral, and septal regions, giving $12 \mathrm{LV}$ segments for analysis. The earliest and latest segments of activation together with the propagation pattern were determined by tracking back in time in the isopotential colour maps. Left bundle activation was evident by the earliest site of activation detected after the His signal in the basal septal region of the left ventricle.

TDI was assessed as previously described. ${ }^{2}$ Myocardial pulse-Doppler velocity signals were reconstituted from the TDI colour images that provided regional myocardial velocity curves. A total of 12 segments, similar to those of non-contact mapping, were analysed. The time to peak myocardial sustained systolic velocity $\left(\mathrm{T}_{\mathrm{S}}\right)$ was measured in each segment by using the onset of the QRS complex as the reference point. The earliest and latest LV segments together with the propagation pattern detected by both methods were compared.

\section{RESULTS}

Preserved left bundle branch activation was detected in three patients. The earliest LV activation was located in the apical anterior and basal septal segments in the other four patients. The earliest LV activation segment by non-contact mapping corresponded well to the earliest $\mathrm{T}_{\mathrm{S}}$ segment by TDI (fig 1).

Two distinct patterns of LV propagation were observed. "Homogeneous" propagation was observed in three patients (type I). There was no acute change in propagation direction during the whole LV depolarisation sequences. Conduction block was observed in the other four patients (type II). There was an acute change in propagation direction and the wavefront split and turned around a region with relatively low voltage in the isopotential map (fig 1 ).

Consistent findings between non-contact mapping and TDI assessment were also observed in detecting the latest segment of activation and systolic movement (fig 1). The latest segments were located in the basal and midlateral area in three patients and in the mid and apical posterior area in four patients.

Abbreviations: LBBB, left bundle branch block; LV, left ventricular, TDI, tissue Doppler imaging; $T_{S}$, time to peak myocardial sustained systolic velocity 

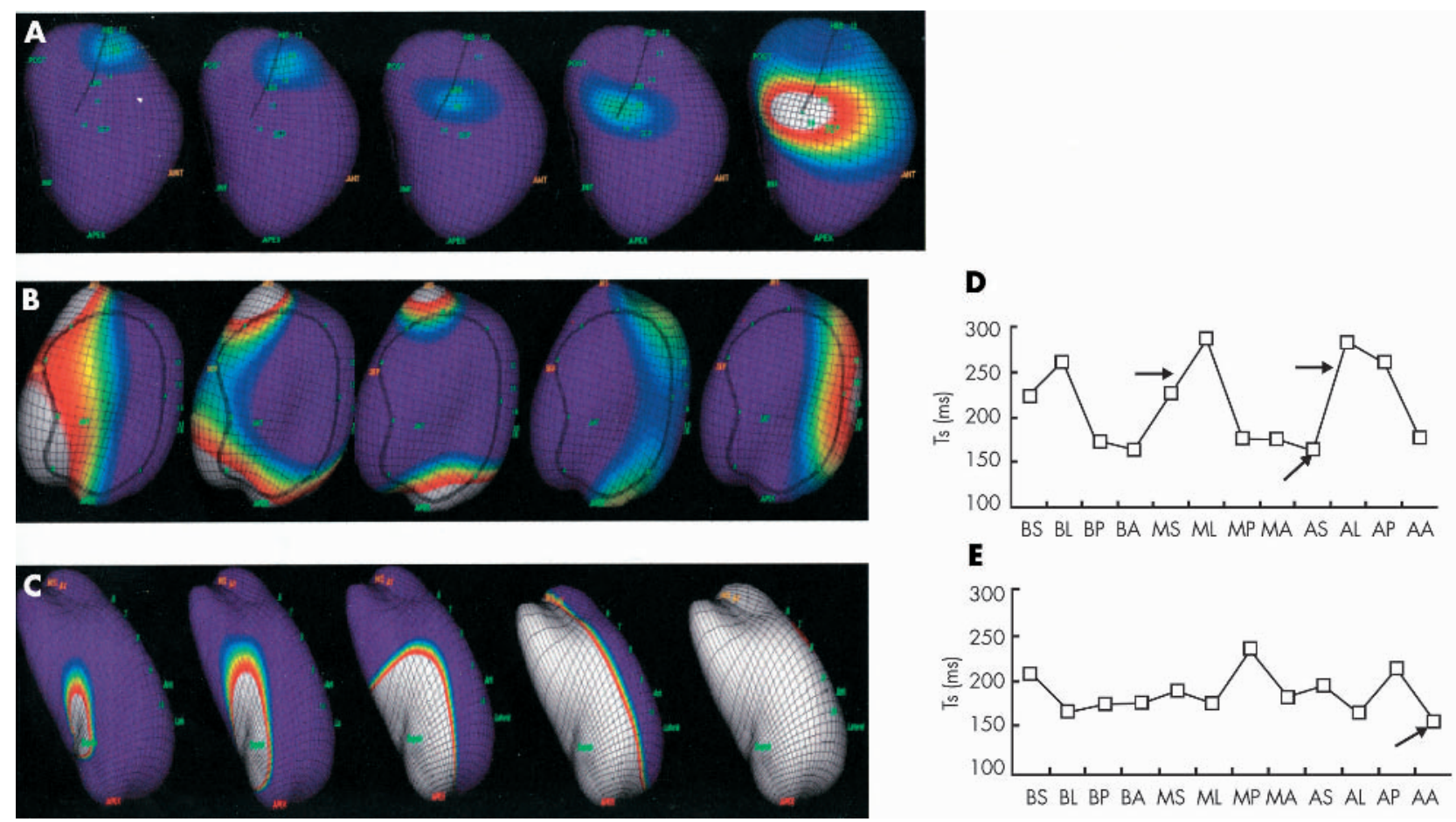

Figure $1(A-C)$ Isopotential maps showing the left ventricular (LV) activation sequences. (A) From left to right: left bundle branch activation. (B) Activation sequences across the LV anterior wall. The depolarisation wavefront splits when approaching the conduction block area (circled by the dark line). The two wavefronts merged in the lateral wall. (C) The endocardial activation originated from the apical anterior area and propagated across the $\mathrm{LV}$ without acute change of direction. (D and E) Corresponding sequences of LV systolic movement in various segments shown by tissue Doppler imaging. (E) The earliest peak systolic movement was located at the apical anterior segment (arrow). There was "homogeneous" propagation across the left ventricle as reflected by relatively minor difference in $T_{S}$ between the LV segments. (D) The earliest peak systolic movement was located at the apical septal area corresponding to left bundle activation (lower arrow). There was a major difference in Ts particularly between septal and lateral LV regions (upper arrows), which was related to the anterolateral conduction block detected by isopotential maps. AA, apical anterior; $\mathrm{AL}$, apical lateral; $A P$, apical posterior segment of $L V$ wall; $A S$, apical septal; BA, basal anterior; $B L$, basal lateral; $B P$, basal posterior; $B S$, basal septal; $M A$, midanterior; ML, mid lateral; MP, mid posterior; MS, mid septal; Ts, time from the onset of QRS complex to peak systolic velocity in each LV segment by tissue Doppler imaging.

\section{DISCUSSION}

The current study found that the LV depolarisation sequences in patients with chronic heart failure and LBBB were variable. Left bundle activation was preserved in three nonischaemic patients despite LBBB. The LV conduction delay may have resulted from either an area of conduction block or slow but homogeneous myocardial propagation. The nature of the conduction block needs to be clarified by future studies. Whether the block was caused by scarred tissue or was functional cannot be determined from the present study. In those with preserved left bundle activation, prolonged LV activation time was likely caused by slow myocardial electrical propagation but not conduction block in one patient. Both types of conduction delay were present in ischaemic and non-ischaemic heart failure. Moreover, the location of the type II block varied between patients. Anterolateral conduction block was observed in two patients and septal block in two patients. For the latest segment of activation, both posterior and lateral locations were observed in this cohort. These findings also suggested that ECG with LBBB could not predict the LV activation sequences. Whether these finding account for the different response to cardiac resynchronisation requires further investigation.

Detailed information about mechanical asynchrony in patients with heart failure and LBBB has been reported previously. ${ }^{2}$ However, information about the LV depolarisation sequences of these patients is lacking. The significance of preserved left bundle activation in these patients is unclear. In two patients with true left bundle blockade, the earliest activation was in apical anterior area rather than directly across the interventricular septum as generally believed. This anomaly may result from subendocardial conduction or other unknown mechanism that could not be detected by noncontact mapping.

The latest LV activation segments were located in either the lateral or posterior regions in this study. This finding is consistent with a study in which TDI was used to assess improved synchronicity after biventricular pacing, which found that systolic motion was latest in the lateral segments. ${ }^{2}$ However, in four patients, the latest segments were located in the posterior region. If sustained clinical improvement is related to pre-excitation of the latest segment, lateral placement of the lead may not always be optimal.

In conclusion, the LV endocardial activation sequences in patients with chronic heart failure and LBBB are variable. Left bundle activation was preserved in some patients with LBBB. TDI correlated well with non-contact mapping for locating the latest segment of activation in these patients and may guide the selection of optimal sites for biventricular pacing.

\section{Authors' affiliations}

JW-H Fung, C-M Yu, G Yip, Y Zhang, H Chan, C-C Kum, JE Sanderson, Division of Cardiology, Department of Medicine and Therapeutics, Prince of Wales Hospital, The Chinese University of Hong Kong, Hong Kong, SAR, China

\section{REFERENCES}

1 Abraham WT, Fisher WG, Smith AL, et al. Cardiac resynchronization in chronic heart failure. N Engl J Med 2002;346:1845-53. 
2 Yu CM, Chau E, Sanderson JE, et al. Tissue Doppler echocardiographic evidence of reverse remodeling and improved synchronicity by simultaneously delaying regional contraction after biventricular pacing therapy in heart failure. Circulation 2002;105:438-45.
3 Butter C, Auricchio A, Stellbrink C, et al. Effect of resynchronization therapy stimulation site on the systolic function of heart failure patients. Circulation 2001;104:3026-9

4 Schilling RJ, Peters NS, Davies DW. Mapping and ablation of ventricular tachycardia with the aid of a non-contact mapping system. Heart 1999:81:570-5.

\section{IMAGES IN CARDIOLOGY}

\section{Neoplastic shock}

A 48 year old woman complained of sudden onset of nausea and vomiting followed by paraesthesia of the tongue and face, dyspnoea, and weakness of her limbs. A severe shock syndrome ensued that necessitated mechanical ventilation and vasopressor support. Laboratory values showed a severe lactic acidosis, hyperglycaemia, and myocardial damage. A global myocardial stunning-like picture without regional motion abnormalities was seen on transoesophageal echocardiography. Aggressive vasopressor and volume therapy was maintained. Nevertheless, the overt left ventricular failure persisted and our patient succumbed to electromechanical dissociation.

Autopsy revealed a right sided retroperitoneal bleeding. A haemorrhagically infarcted phaeochromocytoma with characteristic pale brown cut surface and remnants of the adrenal gland eroded the adjacent vasculature (upper panel). Focal myocardial necrosis, diffuse infiltration of inflammatory cells, accumulation of fatty acids, and contraction band necrosis consistent with catecholamine induced myocarditis was detected (lower panel, haematoxylin-sudan staining). Lipolysis and subsequent accumulation of fatty acids in myocardial cells as well as hyperglycaemia are both metabolic consequences of catecholamine excess. Vasoconstriction and impaired perfusion may account for tissue hypoxia, lactic acidosis, and organ failure. A high degree of clinical alertness is needed to achieve a timely identification of this tumour that has been called the great mimic because of its protean manifestations.

T S Hildebrand B Bockholdt

J S Jürgensen jan_steffen.juergensen@charite.de
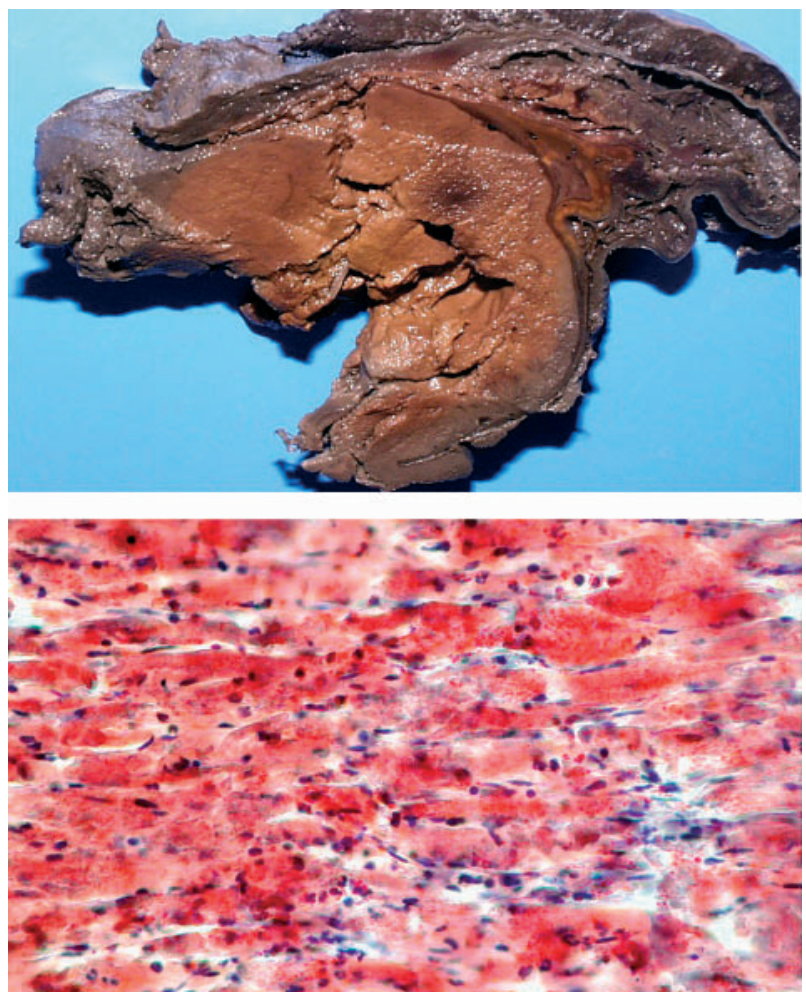

\section{ELECTRONIC PAGES}

\section{Heart Online case reports: www.heartjinl.com}

$\mathrm{T}$

he follow electronic only articles are published in conjunction with this issue of Heart.

\section{Transient abnormal $Q$ waves during exercise electrocardiography}

F F M Alameddine, A M Zafari

Myocardial ischaemia during exercise electrocardiography is usually manifested by ST segment depression or elevation. Transient abnormal Q waves are rare, as Q waves indicate an old myocardial infarction. The case of a patient with exercise induced transient abnormal Q waves is reported. The potential mechanisms involved in the development of such an abnormality and its clinical implications are discussed.

(Heart 2004;90:el) www.heartjnl.com/cgi/content/full/90/ l/el

\section{Paradoxical increase of pulmonary vascular resistance during testing of inhaled iloprost M Emmel, B Keuth, S Schickendantz}

The case of a 14 month old girl with primary pulmonary hypertension treated with domiciliary oxygen is described. After invasive evaluation and testing of nitric oxide with very good response, the testing was repeated to study the effect of inhaled iloprost on pulmonary vascular resistance (PVR). An unexpected and severe increase of PVR was observed, rising from 392 dynes $\cdot s \cdot \mathrm{cm}^{-5}$ with oxygen to a maximum of 1192 dynes $\cdot \mathrm{s} \cdot \mathrm{cm}^{-5}$ with oxygen and iloprost. Underlying ventilatory and technical problems were excluded. While inhaled iloprost has been described to be highly effective in the treatment of primary pulmonary hypertension, the possibility of contrary "paradoxical" reactions in isolated patients is emphasised, with a dramatic increase of PVR and a possible adverse outcome.

(Heart 2004;90:e2) www.heartjnl.com/cgi/content/full/90/1/e2 